\title{
ORIGINAL
}

\section{EVOLUCIÓN DE LAS COBERTURAS VACUNALES ANTIGRIPALES ENTRE 1993-2001 EN ESPAÑA. ANÁLISIS POR COMUNIDADES AUTÓNOMAS}

Elga Mayo Montero (1), Valentín Hernández Barrera (1), Maa José Sierra Moros (2), Isabel Pachón del Amo (2), Pilar Carrasco Garrido (1), Ángel Gil de Miguel (1) y Rodrigo Jiménez García*(1)

(1) Unidad de Docencia e Investigación en Medicina Preventiva y Salud Pública. Facultad de Ciencias de la Salud. Universidad Rey Juan Carlos. Avda de Atenas s/n, Alcorcón 28402 Madrid, Spain.

(2) Ministerio de Sanidad y Consumo. Dirección General de Salud Pública. Subdirección General de Promoción de la Salud y Epidemiología. Po del Prado 18-20, 28014 Madrid, Spain.

\section{RESUMEN}

Fundamento: La gripe es una enfermedad con una elevada morbilidad y que ocasiona un alto coste sanitario, para la que además se bilidad y que ocasiona un alto coste sanitario, para la que ademas se
dispone de una vacuna eficaz. El objetivo de este trabajo es evaluar la evolución de las coberturas vacunales antigripales en España por la evolución de las coberturas vacunales antigip
Comunidades Autónomas entre 1993 y 2001.

Métodos: Se han analizado un total de 42.123 registros de la Encuesta Nacional de Salud (ENS) de los años $1993(\mathrm{n}=21.051)$ y 2001 $(n=21.072)$. Todos ellos corresponden a adultos españoles mayores de 15 años no institucionalizados. Ambas encuestas son representativas a nivel de Comunidad Autónoma.

Resultados: Para el total de la muestra se estima una cobertura vacunal en 1993 de $17,94 \%$ (IC $95 \% 17,42-18,46$ ) y de $19,30 \%$ (18,77-19,83) en 2001. En el modelo de regresión logística, ajustado por edad, género y enfermedad crónica asociada, se observan mejopor edad, género y enfermedad crónica asociada, se observan mejo-
ras significativas en las coberturas del grupo de mayores de 64 años (OR=1,28 IC 95\% 1,10-1,50) para el total de España y para cinco de las 17 Comunidades Autónomas entre 1993 y 2001. Sin embargo, no encontramos cambios significativos en las coberturas para el grupo de menores de 65 años con enfermedad crónica asociada que supone una indicación para la vacunación.

Conclusiones: Tanto en España como en la mayoría de las Comunidades Autónomas entre 1993 y 2001 se observa una ligera pero insuficiente mejón en las coberturas d pero insticien Arupos de riesgo estudiados. Las Comunidades Autónomas muestran enfermedades crónic las complicaciones de la gripe no han mejorado las coberturas de esta vacuna durante el periodo de estudio. La dificultad para mejorar las coberturas en este tipo de pacientes podría hacernos considerar la opción y el beneficio potencial de rebajar la edad limite de las actuales recomendaciones de indicación de vacuna antigripal en España.

Palabras clave: Gripe. Vacunas. Coberturas vacunales. Encuestas de salud.

\section{Correspondencia:}

Rodrigo Jiménez García

Unidad de Docencia e Investigación en Medicina Preventiva y Salud Pública.

Facultad de Ciencias de la Salud. Avda. de Atenas s/n. 28922

Alcorcón. Madrid. España

Tel: 914888853

Correo electrónico: rjimenezg@cs.urjc.es

\section{ABSTRACT}

Evolution of the Degrees of Influenza Vaccine (Flu Shot) Coverage in Spain throughout the 1993-2001 Period. Analysis by Autonomous Communities

Background: Influenza (flu) is a disease involving a high morbidity which takes a high toll on healthcare costs for which there i an effective vaccine available. This study is aimed at evaluating the evolution of the different degrees of influenza vaccine (flu shot) coverage in Spain by Autonomous Communities during the 1993 2001 period.

Methods: A total of 42,123 cases from the National Health Survey (NHS) for the years $1993(n=21,051)$ and 2001 $(n=21,072)$ were analysed, all of which corresponded to non-institutionalised Spanish adults over 15 years of age. Both surveys are representative at the Autonomous Community level.

Results: For the total sample, a vaccine coverage of $17.94 \%(\mathrm{CI}$ $95 \% 17.42-18.46)$ in 1993 and of $19.30 \%$ (18.77-19.83) en 2001 was estimated. In the logic regression model, adjusted by age, sex and related chronic disease, significant improvements were found in the degrees of coverage of individuals over 64 years of age $(\mathrm{OR}=1.28$ CI 95\% 1.10-1.50) for Spain as a whole and for five of the seventeen Autonomous Communities between 1993 and 2001. However, no significant changes were found in the degrees of coverage for the group under age 65 with related chronic disease entailing an indication for being administered the vaccine.

Conclusions: A slight yet insufficient improvement in the degrees of coverage among the high-risk groups studied were found to exist both nation-wide in Spain as a whole as well as in the majority of the both nation-wide in Spain as a whole as well as in the majority of the rage varied greatly among Autonomous Communities. The indivirage varied greatly among Autonomous Communities. The indivi-
dual under age 65 with related chronic diseases heightening the risks dual under age 65 with related chronic diseases heightening the risks
of suffering from flu-related complications have not improved their of suffering from flu-related complications have not improved their
degrees of coverage regarding this vaccine during the period studied. degrees of coverage regarding this vaccine during the period studied. The difficulty involved in improving the degrees of coverage among
this type of patients might lead us to consider the alternative and potential benefit of lowering the age limit of the current influenz vaccine (flu shot) indication-related recommendations in Spain. veys. 


\section{INTRODUCCIÓN}

La gripe constituye una causa importante de morbi-mortalidad en el mundo, provocando entre tres y cinco millones de casos de enfermedad grave y entre 250.000-500.000 muertes al año ${ }^{1}$. Esto sucede pese a la demostrada efectividad de la vacunación antigripal para reducir la enfermedad en sujetos sanos menores de 65 años y para disminuir las complicaciones, las hospitalizaciones y la muerte en sujetos mayores de 65 años y/o con trastornos médicos subyacentes $^{2-4}$.

En España, desde la década de los ochenta, y según los protocolos de vigilancia epidemiológica aprobados por el Consejo Interterritorial del Sistema Nacional de Salud, las indicaciones de la vacunación antigripal incluyen entre otros grupos a todos los sujetos mayores de 65 años y aquellos de menor edad con enfermedades crónicas que les hacen susceptibles de sufrir las complicaciones de la gripe, para todos ellos la administración es gratuita en todas las Comunidades Autónomas (CCAA) ${ }^{5}$. Al igual que en otros países europeos como Alemania, Austria y Hungría, desde la temporada 2003-2004 en Cataluña está indicada la vacuna a partir de los 60 años ${ }^{3,6-9}$. Una de las prioridades en el uso de las vacunas antigripales es el aumento de la cobertura entre las personas con patología de riesgo menores de 65 años ${ }^{4}$.

Los estudios de coberturas vacunales permiten diseñar estrategias adecuadas para mejorar los objetivos, apoyar con datos útiles la toma de decisiones y gestionar los programas de vacunación ${ }^{8-10}$.

Tanto en España como en el resto de Europa los datos sobre coberturas antigripales se centran fundamentalmente en sujetos de edad avanzada ${ }^{11}$. La vacunación antigripal en estas personas presenta coberturas muy heterogéneas, destacando entre las más altas Islandia, Finlandia, Holanda, España y Francia $^{12}$. El cálculo de las coberturas es dis- tinto según el país a considerar; básicamente se basan en las historias clínicas (Dinamarca, Reino Unido y Holanda), certificados de salud (Francia), encuestas poblacionales, o dosis distribuidas por grupo de edad y población susceptible de vacunación (España, Austria, Bélgica y Grecia) ${ }^{13}$. En nuestro país y según encuestas realizadas a nivel nacional, durante los últimos años las coberturas vacunales en el grupo de edad mayor o igual a 65 años son próximas al 50\%, resultados similares a los encontrados en otros países europeos como Italia o Suiza, e inferiores a las obtenidas en Estados Unidos ${ }^{4,11,14,15}$. Las tasas de cobertura vacunal de cada Comunidad Autónoma se recogen de forma habitual para los mayores de 64 años si bien son obtenidos utilizando diferentes fuentes de información ${ }^{16-19}$.

La falta de datos en otros grupos de edad se debe en gran medida a la dificultad para disponer de denominadores sobre personas en las que estaría indicada la vacunación ${ }^{20}$.

Diferentes autores han señalado que las Encuestas Nacionales de Salud son una herramienta útil para estudiar las coberturas vacunales antigripales, los factores asociados a dicha vacunación y la evolución temporal $^{14,15,21-26}$.

El objetivo de este trabajo es evaluar la evolución de las coberturas antigripales en España para cada una de las CCAA a partir de los datos de las Encuestas Nacionales de Salud de España para el periodo trascurrido entre 1993 y 2001. El análisis se realizará por grupos de edad y según la coexistencia de enfermedades concomitantes que constituyen una indicación para la vacunación.

\section{MATERIAL Y MÉTODOS}

Se realiza un estudio transversal acerca de la cobertura de vacunación antigripal en el área geográfica del Estado Español para adultos mayores de 15 años. Se han utilizado 
datos obtenidos de las Encuestas Nacionales de Salud (ENS) realizadas por el Ministerio de Sanidad y Consumo en los años 1993 y $2001^{27,28}$. Estas encuestas se realizan sobre muestras representativas de la población española no institucionalizada y son igualmente representativas a nivel de Comunidad Autónoma. El procedimiento de muestreo es polietápico, estratificado por conglomerados, con selección de las unidades primarias de muestreo (municipios) y de las unidades secundarias (secciones) de forma aleatoria proporcional y de las unidades últimas (individuos) por rutas aleatorias y cuotas de sexo y edad. Las encuestas de estos años precisan ponderación por provincia y Comunidad Autónoma respectivamente.

Se han utilizado un total de 42.123 encuestas, 21.051 y 21.072 para los años 1993 y 2001 respectivamente de todas las Comunidades Autónomas.

Las variables empleadas en nuestro trabajo se recogen de manera idéntica en las dos encuesta empleadas. Como variable dependiente se ha utilizado la respuesta ( si» o «no») a la pregunta, "Querríamos saber si se ha vacunado de la gripe en la última campa$\tilde{n} a »$. Como variables independientes se han analizado otras preguntas realizadas acerca de: presencia de enfermedad crónica asociada (diabetes y/o asma y/o bronquitis crónica y/o enfermedad del corazón), año de la encuesta y variables sociodemográficas (Comunidad Autónoma, edad y género).

Se ha creado la variable «Grupo de riesgo» que incluye a los sujetos con edad mayor o igual de 65 y a los sujetos menores de 65 años que presentan una enfermedad crónica asociada (diabetes y/o asma y/o bronquitis crónica y/o enfermedad del corazón).

Se ha estimado la cobertura de vacuna antigripal en los dos años para cada una de las Comunidades Autónomas por grupos de riesgo, edad y género, mediante el porcentaje de individuos que manifiestan haber sido o no vacunados frente a la gripe en la última campaña con su correspondiente intervalo de confianza al $95 \%$.

Para valorar la evolución de las coberturas vacunales en el periodo 1993-2001 se ha utilizado un modelo de regresión logística multivariante para datos de muestreos complejos utilizando STATA 8.0.

Para cada una de las 17 CCAA se calcula en primer lugar el porcentaje crudo de vacunados y en segundo lugar mediante un OR ajustado por edad, género y presencia de enfermedad crónica, la evolución de la cobertura entre los años 1993 y 2001 tomando como referencia el primero. También se han evaluado los mismos datos sobre evolución temporal estratificando la muestra en función de la pertenencia o no a «grupo de riesgo» en los que estaría indicada la vacunación y ajustando cada grupo por las correspondientes variables.

\section{RESULTADOS}

Se han analizado un total de 42.123 sujetos mayores de 16 años que han contestado a las Encuestas Nacionales de Salud (ENS). El porcentaje de sujetos que respondieron a la pregunta sobre la vacunación antigripal es superior al $98 \%$ en las dos encuestas utilizadas.

La mujeres representan algo mas del 51\% en todas las encuestas y la media de edad se incrementa de 43,35 (DS 18,4) años en 1993 a 45,03 (DS 18,7) años en 2001 a consecuencia del envejecimiento de la población española. En 1993, un 12\% de la muestra tenía por encima de 64 años y un $11 \%$ padecía al menos alguna enfermedad crónica asociada: asma, bronquitis crónica, diabetes y/o enfermedad cardiaca. En 2001, el 20,5\% de los encuestados eran mayores de 64 años y el $13 \%$ presentaban al menos una enfermedad crónica. 
En las tablas 1-4 aparecen las coberturas no ajustadas de vacunación antigripal para el total del país y cada CCAA en los dos años de la ENS. Además figuran los OR ajustados, para cada CCAA y total nacional de la evolución en los dos años teniendo el primero como referencia.

En la tabla 1 se observa que las cifras de cobertura de vacuna de la gripe no aumentan significativamente del año 1993 al 2001 en el total de la muestra (OR=1,02 IC 95\% $0,88-1,08)$, a pesar de que pasen de un $17,94 \%$ (IC $95 \% 17,42-18,46$ ) a un $19,30 \%$ (IC 95\% 18,77-19,83) en el análisis crudo. Sin embargo, al analizar los datos ajustados se observa aumento significativo de la cobertura desde 1993 a 2001 en Aragón, Baleares y Cataluña; mientras detectamos un discreto pero significativo descenso en la C. Valenciana, Extremadura, Navarra y País Vasco. El incremento mayor se produce en Cataluña, comunidad en la cual la probabilidad de estar vacunado ha aumentado desde 1993 a 2001 en un 56\% (OR= 1,56 IC95\% $1,29-1,89)$.

En la tabla 2 se muestran los resultados para el grupo de individuos que son menores de 65 años y que presentan alguna enfermedad crónica asociada. La evolución de la cobertura nacional ajustada por edad y género no presenta cambios significativos para este periodo de tiempo. Sin embargo, aparecen CCAA como Asturias en la que se presenta una mejoría muy importante de la cobertura (OR=3,26; IC 95\% 1,32-8,02). Así mismo, se producen aumentos significativos en Cataluña y Madrid, mientras en La Rioja desciende.

La tabla 3 muestra los resultados obtenidos para el grupo de sujetos de 65 años o más, independientemente de padecer enfermedades asociadas. La mayoría de las CCAA han alcanzado coberturas crudas superiores al 50\% en el año 2001. La frecuencia de individuos que declaran haber recibido la vacuna en este grupo ha mejora- do un $28 \%(\mathrm{OR}=1,28$ IC $95 \%$ 1,10-1,50) a nivel nacional. Se observa al estratificar por CCAA una mejoría significativa de la cobertura en 5 de las 17 CCAA: Andalucía, Aragón, Asturias, Baleares y Cataluña (OR 1,$89 ; 2,29 ; 2,11 ; 1,40$ y 1,96 respectivamente) y por el contrario en Extremadura desciende significativamente su cobertura.

En la tabla 4 se muestran los resultados para los sujetos clasificados como pertenecientes a «grupo de riesgo» que incluye a aquellos con edad mayor o igual de 65 y a los menores de 65 años que presentan una enfermedad crónica asociada (diabetes y/o asma y/o bronquitis crónica y/o enfermedad del corazón). Los porcentajes crudos en el año 2001 indican coberturas alrededor del 50\% en la mayoría de las CCAA. Se detectan mejoras significativas entre el año 1993 y 2001 para el global nacional (OR 1,18 IC 95\% 1,03-1,34), pero tan sólo hay tres CCAA que presentan mejoras significativas Aragón (OR =1,53 IC95\% 1,04-2,25), Asturias $(\mathrm{OR}=1,94 \mathrm{IC} 95 \% 1,12-3,39)$ y Cataluña $(\mathrm{OR}=1,98 \%$ IC95\% 1,43-2,74).

\section{DISCUSIÓN}

La Encuesta Nacional de Salud (ENS), al igual que otras herramientas similares (National Health Interview Survey o Behavioral Risk Factor Surveillance Survey de EEUU), han sido utilizadas en numerosos trabajos para el estudio de problemas relacionados con la salud pública y en concreto para el cálculo de la cobertura vacunal, su evolución y factores asociados ${ }^{15,16,18}$.

El uso de estas encuestas podría suponer una limitación para el estudio por utilizar datos autodeclarados sobre vacunación. Sin embargo, se han comparado este tipo de testimonios directos (auto-declaración) con registros médicos de vacunas, mostrando que los primeros son muy sensibles y poco específicos, y además se ha encontrado una buena concordancia entre ambas fuentes ${ }^{29-31}$.

Rev Esp Salud Pública 2004, Vol. 78, N. ${ }^{\circ} 4$ 
Coberturas globales crudas de vacunacion antigripal por Comunidades Autonomas y total nacional. Evolucion ajustada desde 1993 a 2001

\begin{tabular}{|c|c|c|c|c|c|c|c|c|c|}
\hline \multirow{3}{*}{$\begin{array}{c}\text { Comunidades Autónomas } \\
\text { Andalucía } \\
\end{array}$} & \multicolumn{3}{|c|}{ Año 1993} & \multicolumn{3}{|c|}{ Año 2001} & \multicolumn{3}{|c|}{$\begin{array}{l}\text { OR ajustado de la evolución entre } \\
\qquad 1993-2001\end{array}$} \\
\hline & \multirow{2}{*}{$\begin{array}{c}\text { Cobertura cruda } \\
18,41 \%\end{array}$} & \multicolumn{2}{|c|}{ IC 95\% } & \multirow{2}{*}{$\begin{array}{c}\text { Cobertura cruda } \\
18,32 \%\end{array}$} & \multicolumn{2}{|c|}{ IC 95\% } & \multirow{2}{*}{$\begin{array}{l}\mathbf{O R}^{*} \\
0,93 \\
\end{array}$} & \multicolumn{2}{|c|}{ IC $95 \%$} \\
\hline & & 17,16 & 19,67 & & 17,07 & 19,56 & & 0,78 & 1,09 \\
\hline Aragón & $17,11 \%$ & 14,19 & 20,04 & $24,88 \%$ & 21,53 & 28,23 & $1,51^{* *}$ & 1,19 & 1,91 \\
\hline Asturias & $14,46 \%$ & 11,55 & 17,38 & $19,66 \%$ & 16,44 & 22,88 & 1,23 & 0,88 & 1,72 \\
\hline Baleares & $17,88 \%$ & 14,05 & 21,70 & $21,82 \%$ & 17,69 & 25,94 & $1,24 * *$ & 1,02 & 1,52 \\
\hline Canarias & $13,10 \%$ & 10,81 & 15,38 & $12,44 \%$ & 10,21 & 14,67 & 0,85 & 0,68 & 1,06 \\
\hline Cantabria & $18,25 \%$ & 13,76 & 22,73 & $20,56 \%$ & 15,88 & 25,23 & 0,98 & 0,71 & 1,35 \\
\hline Castilla-La Mancha & $24,18 \%$ & 21,37 & 27,00 & $25,08 \%$ & 22,25 & 27,91 & 0,87 & 0,68 & 1,10 \\
\hline Castilla-León & $22,21 \%$ & 20,00 & 24,43 & $23,26 \%$ & 21,01 & 25,50 & 0,86 & 0,69 & 1,08 \\
\hline Cataluña & $12,35 \%$ & 11,23 & 13,48 & $19,21 \%$ & 17,87 & 20,56 & $1,56^{* *}$ & 1,29 & 1,89 \\
\hline Com.Valenciana & $21,53 \%$ & 19,76 & 23,29 & $18,71 \%$ & 17,04 & 20,37 & $0,73 * *$ & 0,62 & 0,86 \\
\hline Extremadura & $23,53 \%$ & 20,07 & 26,99 & $20,21 \%$ & 16,92 & 23,49 & $0,68^{* *}$ & 0,53 & 0,87 \\
\hline Galicia & $17,52 \%$ & 15,57 & 19,46 & $17,88 \%$ & 15,93 & 19,83 & 0,90 & 0,73 & 1,12 \\
\hline Madrid & $18,49 \%$ & 17,01 & 19,96 & $19,81 \%$ & 18,31 & 21,32 & 0,92 & 0,78 & 1,08 \\
\hline Murcia & $19,05 \%$ & 15,82 & 22,28 & $18,52 \%$ & 15,32 & 21,72 & 0,88 & 0,68 & 1,15 \\
\hline Navarra & $17,07 \%$ & 12,72 & 21,43 & $15,57 \%$ & 11,39 & 19,75 & $0,75^{* *}$ & 0,59 & 0,94 \\
\hline País Vasco & $17,63 \%$ & 15,39 & 19,87 & $16,70 \%$ & 14,51 & 18,88 & $0,70^{* *}$ & 0,51 & 0,97 \\
\hline La Rioja & $21,28 \%$ & 14,52 & 28,03 & $20,00 \%$ & 13,37 & 26,63 & 0,82 & 0,59 & 1,15 \\
\hline TOTAL & $17,94 \%$ & 17,42 & 18,46 & $19,30 \%$ & 18,77 & 19,83 & 1,02 & 0,88 & 1,08 \\
\hline
\end{tabular}

* OR ajustados por edad, sexo y enfermedad crónica asociada

$* *(\mathrm{p}<0,05)$ 


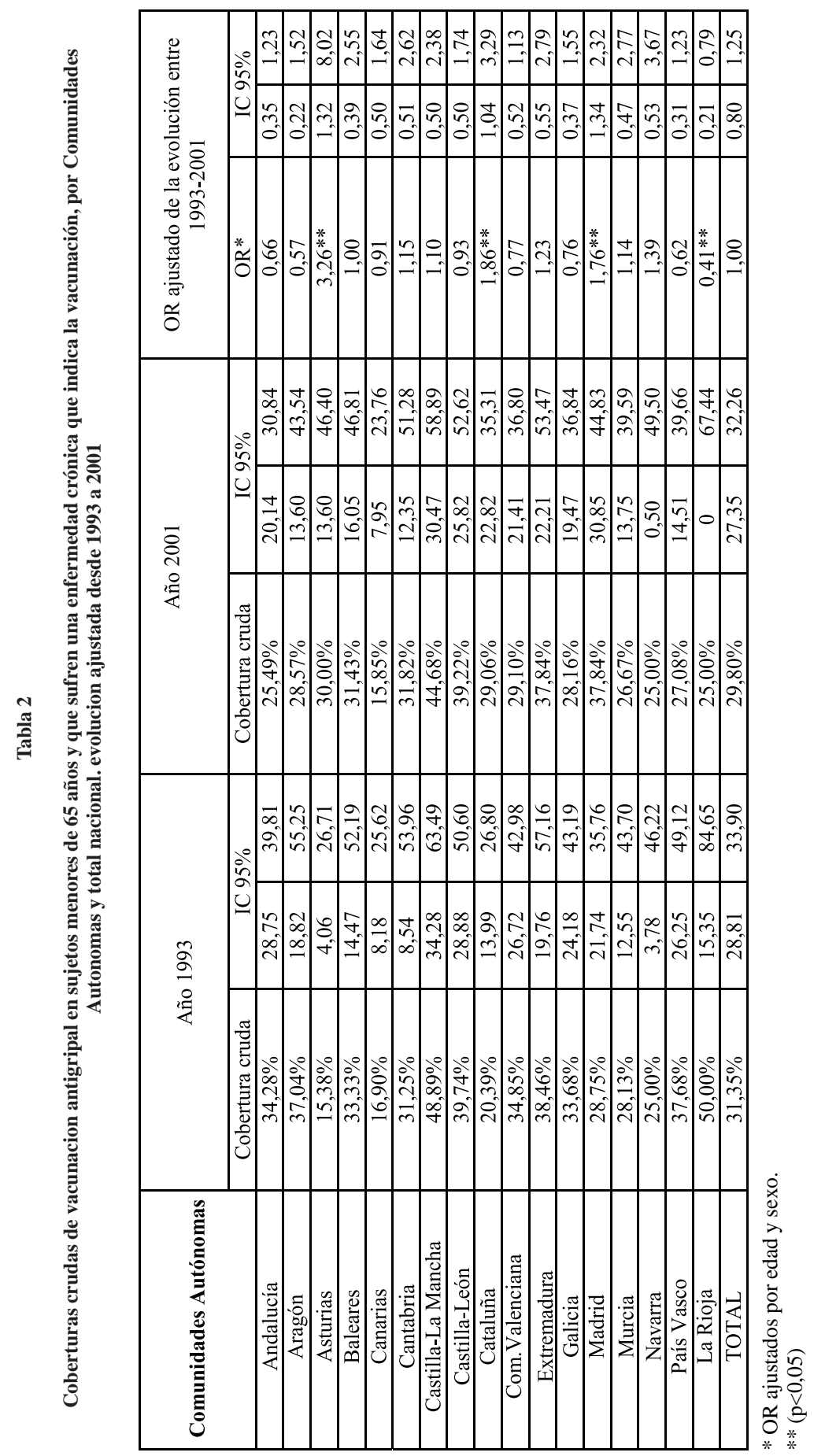


Tabla 3

Coberturas crudas de vacunacion antigripal en sujetos mayores de 64 años por Comunidades Autonomas y total nacional. evolucion ajustada desde 1993 a 2001

\begin{tabular}{|c|c|c|c|c|c|c|c|c|c|}
\hline \multirow{3}{*}{$\begin{array}{c}\text { Comunidades Autónomas } \\
\text { Andalucía } \\
\end{array}$} & \multicolumn{3}{|c|}{ Año 1993} & \multicolumn{3}{|c|}{ Año 2001} & \multicolumn{3}{|c|}{$\begin{array}{l}\text { OR ajustado de la evolución entre } \\
\qquad 1993-2001\end{array}$} \\
\hline & \multirow{2}{*}{$\begin{array}{c}\text { Cobertura cruda } \\
42,60 \%\end{array}$} & \multicolumn{2}{|c|}{ IC $95 \%$} & \multirow{2}{*}{$\begin{array}{c}\text { Cobertura cruda } \\
60,40 \% \\
\end{array}$} & \multicolumn{2}{|c|}{ IC 95\% } & \multirow{2}{*}{$\begin{array}{c}\mathrm{OR}^{*} \\
1,89^{* *}\end{array}$} & \multicolumn{2}{|c|}{ IC 95\% } \\
\hline & & 37,71 & 47,50 & & 56,63 & 64,18 & & 1,38 & 2,58 \\
\hline Aragón & $51,16 \%$ & 40,60 & 61,73 & $71,52 \%$ & 64,48 & 78,56 & $2,29 * *$ & 1,69 & 3,09 \\
\hline Asturias & $40,00 \%$ & 29,59 & 50,41 & $61,31 \%$ & 53,16 & 69,47 & $2,11 * *$ & 1,25 & 3,54 \\
\hline Baleares & $50,00 \%$ & 35,23 & 64,77 & $63,01 \%$ & 51,94 & 74,09 & $1,40^{* *}$ & 1,10 & 1,79 \\
\hline Canarias & $37,31 \%$ & 25,73 & 48,89 & $34,43 \%$ & 26,00 & 42,86 & 1,01 & 0,67 & 1,55 \\
\hline Cantabria & $58,82 \%$ & 42,28 & 75,37 & $62,90 \%$ & 50,88 & 74,93 & 1,34 & 0,81 & 2,13 \\
\hline Castilla-La Mancha & $55,73 \%$ & 47,22 & 64,23 & $62,62 \%$ & 56,01 & 69,23 & 0,87 & 0,61 & 1,24 \\
\hline Castilla-León & $51,96 \%$ & 44,64 & 59,27 & $64,88 \%$ & 59,78 & 69,99 & 1,34 & 0,94 & 1,92 \\
\hline Cataluña & $31,27 \%$ & 26,45 & 36,09 & $54,14 \%$ & 50,42 & 57,86 & $1,96^{* *}$ & 1,52 & 2,53 \\
\hline Com.Valenciana & $51,85 \%$ & 45,19 & 58,52 & $54,46 \%$ & 49,60 & 59,31 & 1,00 & 0,76 & 1,30 \\
\hline Extremadura & $61,97 \%$ & 50,68 & 73,26 & $53,13 \%$ & 44,48 & 61,77 & $0,68^{* *}$ & 0,50 & 0,93 \\
\hline Galicia & $41,99 \%$ & 34,80 & 49,18 & $43,79 \%$ & 38,50 & 49,08 & 0,94 & 0,68 & 1,30 \\
\hline Madrid & $54,92 \%$ & 48,67 & 61,16 & $54,60 \%$ & 50,24 & 58,96 & 0,87 & 0,65 & 1,16 \\
\hline Murcia & $59,62 \%$ & 46,28 & 72,95 & $60,20 \%$ & 50,51 & 69,90 & 0,95 & 0,60 & 1,54 \\
\hline Navarra & $50,00 \%$ & 32,68 & 67,32 & $50,82 \%$ & 38,27 & 63,37 & 1,10 & 0,71 & 1,72 \\
\hline País Vasco & $51,30 \%$ & 42,17 & 60,44 & $50,23 \%$ & 43,63 & 56,82 & 0,84 & 0,59 & 1,21 \\
\hline La Rioja & $52,63 \%$ & 30,18 & 75,08 & $57,58 \%$ & 40,71 & 74,44 & 1,33 & 0,83 & 2,14 \\
\hline TOTAL & $46,49 \%$ & 44,46 & 48,53 & $56,09 \%$ & 54,59 & 57,58 & $1,28 * *$ & 1,10 & 1,50 \\
\hline
\end{tabular}

* OR ajustados por edad, sexo y enfermedad crónica asociada

$* *(\mathrm{p}<0,05)$ 
Coberturas crudas de vacunacion antigripal en sujetos por pertenecer a «grupo de riesgo»\# por Comunidades Autonomas y total nacional. Evolucion ajustada desde 1993 a 2001

\begin{tabular}{|c|c|c|c|c|c|c|c|c|c|}
\hline \multirow{3}{*}{$\begin{array}{l}\text { Comunidades Autónomas } \\
\text { Andalucía }\end{array}$} & \multicolumn{3}{|c|}{ Año 1993} & \multicolumn{3}{|c|}{ Año 2001} & \multicolumn{3}{|c|}{$\begin{array}{l}\text { OR ajustado de la evolución entre } \\
\qquad 1993-2001\end{array}$} \\
\hline & \multirow{2}{*}{$\begin{array}{c}\text { Cobertura cruda } \\
39,11 \%\end{array}$} & \multicolumn{2}{|c|}{ IC $95 \%$} & \multirow{2}{*}{$\begin{array}{c}\text { Cobertura cruda } \\
50,56 \%\end{array}$} & \multicolumn{2}{|c|}{ IC $95 \%$} & \multirow{2}{*}{$\begin{array}{c}\mathrm{OR}^{*} \\
1,40\end{array}$} & \multicolumn{2}{|c|}{ IC $95 \%$} \\
\hline & & 35,43 & 42,79 & & 47,29 & 53,83 & & 0,95 & 2,06 \\
\hline Aragón & $47,79 \%$ & 38,58 & 57,00 & $63,73 \%$ & 56,95 & 70,51 & $1,53 * *$ & 1,04 & 2,25 \\
\hline Asturias & $32,26 \%$ & 24,03 & 40,49 & $56,02 \%$ & 48,47 & 63,57 & $1,94 * *$ & 1,12 & 3,39 \\
\hline Baleares & $44,12 \%$ & 32,32 & 55,92 & $52,78 \%$ & 43,36 & 62,19 & 1,11 & 0,82 & 1,50 \\
\hline Canarias & $27,01 \%$ & 19,57 & 34,44 & $26,60 \%$ & 20,52 & 32,68 & 0,74 & 0,50 & 1,11 \\
\hline Cantabria & $50,00 \%$ & 36,14 & 63,86 & $54,12 \%$ & 43,52 & 64,71 & 0,84 & 0,53 & 1,32 \\
\hline Castilla-La Mancha & $53,98 \%$ & 46,61 & 61,34 & $59,29 \%$ & 53,23 & 65,34 & 1,01 & 0,72 & 1,44 \\
\hline Castilla-León & $48,25 \%$ & 42,14 & 54,36 & $61,50 \%$ & 56,65 & 66,35 & 1,19 & 0,79 & 1,78 \\
\hline Cataluña & $28,01 \%$ & 24,10 & 31,92 & $48,54 \%$ & 45,26 & 51,82 & $1,98^{* *}$ & 1,43 & 2,74 \\
\hline Com.Valenciana & $45,53 \%$ & 40,29 & 50,77 & $48,14 \%$ & 43,92 & 52,36 & 0,86 & 0,68 & 1,09 \\
\hline Extremadura & $55,67 \%$ & 45,78 & 65,56 & $49,39 \%$ & 41,74 & 57,04 & 0,75 & 0,55 & 1,04 \\
\hline Galicia & $39,13 \%$ & 33,37 & 44,89 & $40,14 \%$ & 35,56 & 44,71 & 0,93 & 0,64 & 1,34 \\
\hline Madrid & $44,67 \%$ & 39,81 & 49,52 & $50,07 \%$ & 46,33 & 53,82 & 1,14 & 0,95 & 1,39 \\
\hline Murcia & $47,67 \%$ & 37,12 & 58,23 & $49,65 \%$ & 41,46 & 57,85 & 0,95 & 0,58 & 1,55 \\
\hline Navarra & $41,30 \%$ & 27,08 & 55,53 & $46,58 \%$ & 35,13 & 58,02 & 0,94 & 0,66 & 1,33 \\
\hline País Vasco & $46,20 \%$ & 38,99 & 53,40 & $46,10 \%$ & 40,14 & 52,05 & 0,83 & 0,50 & 1,39 \\
\hline La Rioja & $50,00 \%$ & 30,78 & 69,22 & $55,26 \%$ & 39,45 & 71,07 & 0,91 & 0,60 & 1,39 \\
\hline TOTAL & $36,36 \%$ & $7,94 \%$ & 42,72 & $46,67 \%$ & 48,50 & 51,13 & $1,18^{* * *}$ & 1,03 & 1,34 \\
\hline
\end{tabular}

\# «grupos de riesgos» incluye a los sujetos con edad igual o mayor de 65 años y a los menores de 65 años que presentan una enfermedad crónica asociada (Diabetes mellitus, y/o asma, y/o bronquitis crónica, y/o enfermedad del corazón).

* OR ajustados por edad y sexo.

$* *(\mathrm{p}<0,05)$ 
La tasa de respuesta en la pregunta sobre ser vacunado frente a la gripe ha sido muy elevada en todos los años estudiados lo que puede hacer poco probable la existencia de un sesgo de selección.

La prevalencia de enfermedades crónicas estimada en la ENS para los dos años analizados aumenta de un $11 \%$ a $13 \%$, lo cual se puede explicar por el envejecimiento de la población española, y es similar a la encontrada en otros estudios. Mediante la Encuesta de Salud de Cataluña realizada en 2002 se estimó una prevalencia de factores de riesgo (enfermedades cardiacas, bronquitis crónica, asma y/o diabetes) del $15 \%$ en los mayores de 15 años de esta Comunidad17. En EEUU se estima que con la edad aumenta la proporción de individuos a riesgo de un $11 \%$ en adultos de 18-49 años a un 24-29\% entre los 50 a 64 años ${ }^{4}$. Russell et al en un trabajo realizado en Alberta incluyen como sujetos con enfermedades susceptibles de vacunación las mismas que nuestro estudio, obteniendo una prevalencia para el grupo de 5064 años del $22,8 \%$, pero señalan que no incluir otras enfermedades indicativas de vacunación (inmudeficiencias, neuropatías etc) podría subestimar el porcentaje de sujetos con indicación de la vacuna, a pesar de tener éstas una importancia proporcionalmente menor que las si incluidas por lo que considera que la magnitud del error será pequeña. ${ }^{20}$.

Las tasas de cobertura observadas en este estudio para los mayores de 16 años a nivel nacional entre 1993 y 2001 no presentan mejorías significativas, y por CCAA presentan aumentos significativos tan sólo Aragón, Baleares y Cataluña. Obtenemos un 19,30\% de cobertura en 2001, este resultado es similar al dato de la utilización de la vacuna estimado a partir del número de dosis de vacuna distribuidas en España por cada 1000 sujetos de población general que en el año 2000 ascendió a $178^{7}$. Para la temporada 20002001 se realizó en Cataluña una encuesta telefónica que estimó una cobertura antigri- pal de $24,6 \pm 1,4 \%$ para todas las edades, resultado ligeramente superior al calculado a partir de la ENS del $2001(19,21 \%)^{17}$.

Existen pocos estudios sobre cobertura obtenidos en el grupo de sujetos jóvenes (menores de 65 años) que se beneficiarían de la vacuna antigripal por tener una enfermedad crónica asociada, fundamentalmente debido al desconocimiento de los denominadores para calcular dichas coberturas ${ }^{20}$. Los porcentajes de vacunación que obtenemos en nuestro estudio en estos sujetos no superan el 30\%. Además, no detectamos mejorías significativas en este periodo para el conjunto de España que a diferencia de otros países si mejoran. En EEUU en la temporada 2000-2001 en el grupo de 18 a 64 años con factores de riesgo asociados se calculó una cobertura de $29 \%$, similar a nuestro trabajo 32. Sin embargo, en la NHIS del año 2002 las coberturas vacunales entre sujetos con factores de riesgo fueron de $23,1 \%$ entre $^{18}$ y 49 años y $43,6 \%$ para los entre 50 y 64 años, muy por encima de las que nosotros calculamos en 2001 para estos mismos grupos $(15,3 \% \text { y } 21,5 \%)^{4}$. En Dinamarca la cobertura entre los adultos jóvenes con enfermedades crónicas es del $33 \%{ }^{12}$.

La Organización Mundial de la Salud, en su Consejo Ejecutivo de noviembre de 2002, instó a los Estados Miembros a la aplicación de estrategias para aumentar las coberturas en los grupos de alto riesgo, incluidas las personas de edad avanzada y los que padecen patologías crónicas, con el fin de conseguir para el año 2010 una cobertura de al menos el $75 \%$ en las personas de edad avanzada.

En este estudio hemos detectado mejorías significativas en el periodo estudiado en coberturas de mayores de 64 años para el total nacional $(46,49 \%$ a $56,09 \%$; OR ajustado 1,28). Los resultados notificados por Estados Unidos sobre coberturas antigripales para este grupo han mejorado linealmente cada año desde 1989 hasta 1997 estabili- 
zándose hasta 1999 y descendiendo en 2001, la NHIS del 2002 obtiene de nuevo mejorías $(65,6 \%)^{4,33,34}$. En Europa los resultados para la temporada 2000-2001 oscilan desde un $15 \%$ en Rumania a un $81 \%$ en Holanda 12. Al igual que en España en otros países se observa un incremento en las coberturas antigripales en mayores de 64 años ${ }^{7,12}$.

Para mayores de 64 años, entre CCAA se observan mejorías muy significativas en este periodo en Asturias, Cataluña y Madrid, pero hay que señalar que las dos primeras partían de unas coberturas bajas en el año 1993 (15,38\% y 20,39\% respectivamente).

En algunos estudios se han realizado cálculos puntuales de cobertura para algunas CCAA con herramientas diferentes a la ENS y con otra metodología. La información aportada mediante este tipo de encuestas parece obtener coberturas superiores a las obtenidas mediante los datos de auto-declaración de la ENS, sin embargo coinciden en el hecho de encontrar coberturas superiores en los grupos de mayor edad. En el año 1999 se realizó un estudio ecológico en la Comunidad Valenciana en el cual se calculaba una cobertura del $70,7 \%$ para la temporada 9697 en sujetos mayores de 64 años 19. Por otro lado, el Ministerio de Sanidad y Consumo facilita estimaciones de cobertura vacunal a partir de las vacunas administradas a mayores de 64 años (disponibles en: www.msc.es). Estos datos estimados desde la temporada 1996-1997 hasta la 2002-2003 $(62,7 \%, 66,8 \%, 63,5 \%, 59,8 \%, 61,5 \%$, $61,9 \%$ y $67,2 \%$ ) demuestran un ligero aumento de las coberturas en el global de España. El rango entre CCAA oscila en la última temporada disponible entre un 39,3 en Canarias y un $86,9 \%$ en Andalucía, y en los datos obtenidos en la ENS del año 2001 esta oscilación está entre el 34,43\% de Canarias y el 71,52\% de Aragón.

La cobertura calculada en todos los grupos en los que está indicada la vacuna (Grupo de riesgo), ha pasado de 36,36\% en 1993 a $46,67 \%$ en 2001 , hemos encontrado por lo tanto mejorías significativas en este periodo $(\mathrm{OR}=1,18$ IC95\% 1,03-1,34). El análisis en detalle de los datos señalaría que el incremento de coberturas dentro de este grupo de riesgo se ha producido fundamentalmente a expensas de los sujetos con indicación de la vacuna por tener una edad superior a 64 años y que como se ha señalado previamente los porcentajes de vacunación no mejoran significativamente entre 1993 y 2001 en el total de la población ni en el grupo de menores de 65 .

Para concluir, detectamos en primer lugar una mejoría de coberturas en los mayores de 64 años para el global de España y parte de las CCAA, pero quedan descuidados el grupo de menores de esa edad que se beneficiaría de la vacuna por presentar enfermedades crónicas asociadas. Una razón podría ser que las campañas de vacunación no se estén dirigiendo a este grupo con la misma intensidad o que si se hacen no estén resultando tan eficaces. Además hay evidencias que apuntan a que las estrategias basadas en la edad son más exitosas para mejorar coberturas vacunales que las dirigidas a pacientes seleccionados por su condición médica de riesgo $0^{4,35}$. En segundo lugar, las coberturas dispares encontradas entre CCAA parece indicar que pueden existir diferencias en las estrategias y las campañas de vacunación aplicadas en cada zona obteniendo así mejores tasas de vacunación en unas CCAA que en otras.

En el año 2000 el ACIP (Advisory Comité of Immunization Practices) modificó las recomendaciones para la vacunación antigripal reduciendo la edad de vacunación universal de 64 a 50 años con el fin de mejorar las tasas en el grupo más joven que tiene enfermedades crónicas ${ }^{32}$. En España sólo en Cataluña, como en otros países Europeos, se ha descendido la edad de indicación de la vacuna a partir de los 60 años. Aún es pronto para valorar si medidas como ésta serán eficaces en la mejora de coberturas y sería deseable realizar estudios dirigidos a valorar 
estas estrategias tanto a nivel sanitario como económico.

\section{BIBLIOGRAFIA}

1. Influenza: Report by the WHO Secretariat, World Health Assembly - May 2003 http://www.who. int/gb/EB_WHA/PDF/WHA56/ea5623.pdf

2. Vu T, Farish S, Jenkins M, Kelly H. A meta-analysis of effectiveness of influenza vaccine in persons aged 65 years and over living in the community. Vaccine. 2002;20(13-14):1831-6.

3. Nichol KL. The efficacy, effectiveness and costeffectiveness of inactivated influenza virus vaccines. Vaccine 2003; 21: 1769-1775

4. Centers for Disease Control and Prevention. Prevention and control of influenza: recommendations of the Advisory Committee on Immunization Practices. MMWR 2004;53:[1-40].

5. Costa Tadeo X, Navarro Aznarez G, Campos Abellana C, Esporrin Bosque C, Romero Ruiz AI. Evaluacion del no cumplimiento de un programa de vacunación antigripal. Aten Primaria. 1991; 8(7):544-6.

6. Generalidad de Cataluña. Departamento de Sanidad y Seguridad Social.Dirección General de Salud Pública. Guía técnica de la vacuna antigripal Campaña 2003-2004. http://www.gencat.net/sanitat/ depsan/units/sanitat/pdf/esguiavagripcas.pdf.

7. Van Essen G.A, Palache A..M., Forleo, D.S. Fedson. Influenza vaccination in 2000: recommendations and vaccine use in 50 developed and rapidly developing countries. Vaccine 21 (2003) 17801785.

8. Bridges CB,Thompson WW, Meltzer MI, Reeve GR, Talamonti WJ et al. Effectiveness and costbenefit of influenza vaccination of healthy working adults:A randomized controlled trial.JAMA 2000; 284:1661-5

9. T. M. Govaert; C. T. Thijs; N. Masurel; M. J. Sprenger; G. J. Dinant; J. A. Knottnerus.The efficacy of influenza vaccination in elderly individuals.A randomized double-blind placebo-controlled trial.JAMA 1994; 272:166-5.

10. Guerin N. Assessing immunization coverage: how and why? Vaccine 1998;16; Suppl:S81-3.
11. LeBaron CW, Chaney M, Baughman AL, Dini EF, Maes E, Dietz V, Bernier R. Impact of measurement and feedback on vaccination coverage in public clinics, 1988-1994. JAMA 1997; 277(8):631-35.

12. Kroneman M, Paget WJ, van Essen GA. Influenza vaccination in Europe: an inventory of strategies to reach target populations and optimise vaccination uptake.EuroSurveill.2003Jun;8(6):130-8.

13. Ambrosch F, Fedson D Influenza vaccination in 29 countries. An update to 1997. Pharmaeconomics1999;16(Supl1):47-54.

14. Navarro JA, Bernal Gonzalez PJ. Calendarios, coberturas vacunales y enfermedades inmunoprevenibles en países de Europa Occidental.Vacunas 2003; 4:188- 194.

15. Jimenez R, Larrauri A, Carrasco P, Esteban J, Gomez-Lopez LI, Gil A. Influenza coverages in Spain and vaccination-related factors in the subgroup aged 50-64 years. Vaccine. 2003 Sep 8;21(25-26):3550-5.

16. Sarriá A; Timoner J. Determinantes de la vacunación de la gripe en personas mayores de 65 años. REv Esp Salud Pública 2002; 76:17-26.

17. Batalla J, Urbitzondo L, Martinez M, Batista JM, Parrón I;Taberner, Salleras Ll. La cobertura de la vacunación antigripal en Cataluña.Vacunas 2001; 2:130-134.

18. Domínguez A, Juncá R, Tresserres, J, Batalla J, Salleras L.Influenza vaccination: the prevalence of risk factors in persons aged 60 years and more in Catalonia Vacunas 2003;4:114-8.

19. Puig-Barberá J; Ors Zarzoso P; Vilches Peña C; Lloria Paes F; Impacto de las distintas estrategias en las tasas de vacunación antigripal en ancianos. At Primaria 1996;6:339-345.

20. Russell ML. Denominators for estimation of influenza vaccine coverage among high risk persons aged 15 to 64 years. Can J Public Health 1996;87(5):301-4.

21. Pleis JR, Gentleman JF. Using the National Health Interview Survey: time trends in influenza vaccinations among targeted adults. Eff Clin Pract. 2002 May-Jun;5(3 Suppl):E3

22. Nelson DE, Powell-Griner E, Town M, Kovar MG.A comparison of national estimates from the National Health Interview Survey and the Behavio- 
ral Risk Factor Surveillance System. Am J Public Health. 2003 Aug;93(8):1335-41.

23. Kamal KM, Madhavan SS, Amonkar MM. Determinants of adult influenza and pneumonia immunization rates. J Am Pharm Assoc (Wash DC). 2003 May-Jun;43(3):403-11

24. Singleton JA, Greby SM, Wooten KG, Walker FJ, Strikas R. Influenza, pneumococcal, and tetanus toxioid vaccination of adults-United States, 1993-7.MMWR CDC Surveill Summ. 2000 Sep 22;49(9):39-62..

25. Janes GR, Blackman DK, Bolen JC, Kamimoto LA, Rhodes L, Caplan LS, et al. Surveillance for use of preventive health-care services by older adults, 1995-1997. MMWR CDC Surveill Summ. 1999 Dec 17;48(8):51-88.

26. Ford ES, Mannino DM, Williams SG. Asthma and influenza vaccination: findings from the 19992001 National Health Interview Surveys. Chest. 2003 Sep;124(3):783-9.

27. Ministerio de Sanidad y Consumo, Subdirección General de Epidemiología, Promoción y Educación para la Salud. Encuesta Nacional de Salud de España 1993 [Spanish National Health Survey 1993].Madrid (Spain), 1995.

28. Ministerio de Sanidad y Consumo, Subdirección General de Epidemiología, Promoción y Educación para la Salud. Encuesta Nacional de Salud de España 2001 [Spanish National Health Survey 2001].Madrid (Spain), 2003.

29. Mac Donald R, Baken L, Nelson A, Nichol KL. Validation of sel-reported of influenza and pneu- mococcal vaccination status in elderly outpatients. Am J Prev Med 1999;16(3):67-72.

30. Hutchinson BG. Measurement of influenza vaccination status of the elderly by mailed questionnaire:response rate, validity and cost. Can J Public $1989 ; 80(4): 271-5$

31. Zimmerman RK, Raymund M, Janosky JE, Nowalk MP, Fine MJ. Sensitivity and specificity of patient self-report of influenza and pneumococcal polysaccharide vaccinations among elderly outpatients in diverse patient care strata. Vaccine. 2003 Mar 28;21(13-14):1486-91.

32. Centers for the Disease Control and Prevention Prevention and control of influenza recommendations of the Advisory Committee on Immnization Practices. MMWR Morb Mortal Wkly Rep 2000;49(RR-3):6.

33. CDC. Racial/ethnic disparities in influenza and pneumococcal vaccination levels among persons aged $>$ or $=65$ years-United States, 1989-2001 MMWR Morb Mortal Wkly Rep. 2003 Oct 10;52(40):958-62.

34. CDC. Influenza and pneumococcal vaccination levels among persons aged $>$ or $=65$ years-United States, 2001. MMWR Morb Mortal Wkly Rep. 2002;5:1019-24

35 Honkanen PO, Keistinen T, Kivela SL The impact of vaccination strategy and methods of information on influenza and pneumococcal vaccination coverage in the elderly population. Vaccine. 1997; 15(3):317-20. 